\title{
Primary Squamous Cell Carcinoma of the Liver is Rare but Hostile: Case Series and Comprehensive Review of the Literature
}

This article was published in the following Dove Press journal: Cancer Management and Research

\section{Junjuan Xiao \\ Lin $\mathrm{Ma}$ \\ Junwei Li \\ Beibei Yin \\ Jing Liang (D) \\ Jun Wang}

Department of Oncology, The First Affiliated Hospital of Shandong First Medical University, Jinan, People's Republic of China
Correspondence: Jun Wang

Department of Oncology, The First Affiliated Hospital of Shandong First

Medical University, No. 16766, Jingshi

Road, Jinan 250014, People's Republic of

China

Tel +86-531-8926-8839

Email ggjun2005@।26.com

Jing Liang

Department of Oncology, The First Affiliated Hospital of Shandong First

Medical University, No. 16766, Jingshi

Road, Jinan 250014, People's Republic of

China

Tel +86-53I-8926-93I6

Email liangjing053I@I63.com

\begin{abstract}
Primary squamous cell carcinoma (SCC) of the liver is an uncommon cancer type. Only dozens of such cases have been reported in the literature. We reviewed three cases with primary SCC of the liver in a single center from January 2013 to October 2019. One case was positive for hepatitis B infection and simultaneously diagnosed with sigmoid adenocarcinoma and liver cyst. The second patient presented with hepatolithiasis. The remaining one had no history of prior liver insult, hepatic infection or any pre-existing hepatic cysts. Two cases had a long survival of more than one year through chemotherapy, or radical surgery plus transarterial chemoembolization. We also found 25 patients with primary hepatic SCC in the Surveillance, Epidemiology and End Results (SEER) database from 1997 to 2016. The median age was 67 years (range 33-87 years). The median overall survival and disease-specific survival were 7.7 months (range 0.0-76.0 months) and 2.0 months (range 0.0-20.0 months), respectively. Furthermore, patients receiving surgery had a longer median OS (20.0 versus 6.0 months, $P=0.016)$ and DSS (48.0 versus 8.0 months, $P=0.03)$ than those receiving palliative treatment. Only $20 \%$ of all cases survived for more than a year. Although primary SCC of the liver has an unfavorable prognosis, radical surgery and systematic treatment might be helpful for clinical management.
\end{abstract}

Keywords: primary squamous cell carcinoma, liver, survival, surgery, chemotherapy, SEER

\section{Introduction}

Primary squamous cell carcinoma (SCC) of the liver is a rare and easily misdiagnosed disease. ${ }^{1}$ Only dozens of such cases have been reported in the literature since the 1970s. ${ }^{1-10}$ The carcinogenesis and pathogenesis of primary hepatic SCC has not been elucidated to date, but this disease is regularly considered to be associated with hepatic teratoma, congenital cyst of the liver or the long-term inflammation or metaplasia of biliary epithelial cells. ${ }^{3}$ Although some cases with the primary SCC of the liver have been successfully managed with initial surgery and chemotherapy, there is no standard care for this disease. ${ }^{2,6}$ As an aggressive cancer, the prognosis of this tumor is extremely unfavorable with an overall survival (OS) of less than 12 months. ${ }^{1-10}$ In this retrospective study, we reported three cases of primary SCC of the liver in a single center from January 2013 to October 2019 and analyzed their clinical and pathological characteristics, treatment and prognosis. We also reviewed all cases with the primary SCC of the liver from the Surveillance, Epidemiology and End Results (SEER) database from 1997 to 2016. 


\section{Patients and Data}

\section{Patients}

The retrospective case series study included three pathologically confirmed primary SCC of the liver at The First Affiliated Hospital of Shandong First Medical University from January 2013 to October 2019. These primary SCC of the liver account for $0.5 \%$ of all primary hepatic malignancies including 495 patients with hepatocellular carcinoma, 103 with intrahepatic cholangiocarcinoma, and one with adenosquamous carcinoma of the liver. A total of 78 pathologically confirmed patients with metastatic SCC of the liver also were diagnosed during the same period. The clinical data including clinical symptoms, signs, pathological diagnosis, radiological findings, laboratory analyses, treatments, and outcome were obtained by local investigators from the hospital's electronic medical records according to previously designed standardized data collection forms. Pathological diagnosis including immunohistochemistry (IHC) was independently reviewed by two pathologists. Written informed consent for publication was provided by all patients, which was in accordance with the Helsinki Declaration. The approval was obtained from the independent research ethics of The First Affiliated Hospital of Shandong First Medical University (NO: YXLL-KY-2020007) to publish the case details and conduct this research.

We also investigated all primary SCC of the liver in SEER database released in November 2019, which includes data from 18 population-based registries (1976-2016) covering approximately $28 \%$ of cancer patients in the United States. Tumor location, grade, and histology were recorded according to the International Classification of Diseases for Oncology Version 3 (ICD-O-3). The inclusion criteria that were used to identify eligible patients included: age between 18 and 79 years, primary tumor location of the liver (ICD-O-3 site code C22), only one primary site or more primaries, pathologically confirmed squamous cell carcinoma (8070/3, 8071/3, 8072/3, 8073/3, and 8076/3), stages I-IV tumors according to the American Joint Committee on Cancer (AJCC) staging system, 7th version. Finally, 24,508 cases with squamous cell carcinoma and 724,508 cases with primary hepatic malignancy were identified from the SEER database.

\section{Results}

\section{Case Presentation from the Single Center Case 1}

A 79-year-old man was initially admitted to The First Affiliated Hospital of Shandong First Medical University in February 2012, complaining of the loss of appetite and weakness for nearly 3 months. Twenty years ago, he was diagnosed with sigmoid adenocarcinoma and received surgery and postoperative adjuvant chemotherapy. He had no history of any hepatitis virus infection. He had a long history of alcohol consumption, but not gastrointestinal bleeding. On admission, the liver was not touched by physical examination. The platelet count (PLT), prothrombin time (PT), activated partial thromboplastin time (APTT), and liver function tests including alanine aminotransferase (ALT), aspartate transaminase (AST), total bilirubin (TBIL), and direct bilirubin (DBIL) were normal (Table 1). He also had normal levels of alpha fetoprotein (AFP) and SCC antigen, but the levels of cytokeratin 19 fragment (CYFRA21-1) and carcinoembryonic antigen (CEA) were elevated. Computed tomography (CT) revealed multiple low-density lesions with unclear boundary and mild to moderate enhancement in the liver accompanied by liver cysts and gallbladder stones, without portal vein invasion or splenomegaly (Figure $1 \mathrm{~A}$ and $\mathrm{B}$ ). Ultrasonography (US)-guided liver biopsy showed a pathological diagnosis of SCC by hematoxylin-eosin (HE) staining. IHC analysis showed that CK7, hepatocyte and CK19 staining were negative but p63 expression was positive (Figure 1C-E). Ventana IHC analysis (Clone NO. SP263) indicated that PD-L1 expression on tumor cells and tumor-associated immune cells was negative (Figure $1 \mathrm{~F}$ and $\mathrm{G})$. Given that no lesions in the lungs, gastrointestinal tract or other organs were observed, primary SCC of the liver was diagnosed. The patient did not receive surgery, radiotherapy, transarterial chemoembolization (TACE), or tyrosine kinase inhibitor treatment. He was treated with SOX chemotherapy regimen (Oxaliplatin $200 \mathrm{mg}$, day 1, and S-1 $50 \mathrm{mg}$ twice per day, d1-14, q21d) for 4 cycles. However, the patient stopped his therapy 3 months later. Subsequently, his disease progressed, and he rejected any anticancer therapies and died at the 20th month of the initial diagnosis.

\section{Case 2}

A 47-year-old man complained of pain in the right upper region of the abdomen after drinking in October 2016. He had a history of hepatitis $\mathrm{B}$ but not $\mathrm{C}$ virus infection. He also had a long history of alcohol consumption but denied gastrointestinal bleeding history. Physical examination revealed a deep tenderness in his upper abdomen. His blood routine examination and coagulation function were normal. Liver function test showed an obvious increase in ALT, AST but 
Table I Summary of three Patients with SCC of the Liver in Our Hospital

\begin{tabular}{|c|c|c|c|}
\hline & $\begin{array}{l}\text { Patient } \\
\text { I }\end{array}$ & Patient 2 & $\begin{array}{l}\text { Patient } \\
3\end{array}$ \\
\hline Gender & Male & Male & Female \\
\hline Age, years & 79 & 47 & 79 \\
\hline Alcohol consumption & + & + & - \\
\hline $\mathrm{HbsAg}$ & - & + & - \\
\hline HCV-Ab & - & - & - \\
\hline Liver cyst & + & - & - \\
\hline Hepatolithiasis & + & + & - \\
\hline $\begin{array}{l}\text { Gastrointestinal } \\
\text { hemorrhage }\end{array}$ & - & - & - \\
\hline PLT & Normal & Normal & Normal \\
\hline AST/ALT, U/L & $\begin{array}{l}25.3 / \\
22.8\end{array}$ & $673 / 867$ & $\begin{array}{l}17.8 / \\
21.3\end{array}$ \\
\hline TBIL/DBIL, umol/L & $7.7 / 3.7$ & 15.7/7.5 & $9.6 / 5.0$ \\
\hline PT/APTT, second & $\begin{array}{l}10.2 / \\
29.4\end{array}$ & $10.0 / 24$ & $\begin{array}{l}11.8 / \\
26.5\end{array}$ \\
\hline AFP/CEA & $1.2 / 9.38$ & $3.34 / 1.55$ & $\begin{array}{l}0.78 / \\
1.82\end{array}$ \\
\hline SCC/CYFRA2I-I & $5.0 / 86.3$ & - & $\begin{array}{l}14.8 / \\
14.9\end{array}$ \\
\hline Tumor size, cm & 4.0 & 10 & 7.5 \\
\hline Tumor number & Multiple & Single & Single \\
\hline Tumor location & $\begin{array}{l}\text { Whole } \\
\text { liver }\end{array}$ & $\mathrm{VI} / \mathrm{VII}$ & $\begin{array}{l}\text { Right } \\
\text { lobe }\end{array}$ \\
\hline Portal vein invasion & - & Unknown & - \\
\hline Splenomegaly & - & Unknown & - \\
\hline Surgery & - & Hemihepatectomy & - \\
\hline Chemotherapy & + & + & - \\
\hline Radiotherapy & - & - & - \\
\hline $\begin{array}{l}\text { Transarterial } \\
\text { chemoembolization }\end{array}$ & - & + & - \\
\hline Targeted therapy & - & - & Anlotinib \\
\hline Survival, months & 20 & 17 & 2 \\
\hline Outcome & Dead & Alive & Dead \\
\hline
\end{tabular}

Abbreviations: $\mathrm{HBsAg}$, hepatitis $B$ surface antigen; $\mathrm{HCV}-\mathrm{Ab}$, hepatitis $\mathrm{C}$ virus antibody; PLT, platelet count; AST, aspartate transaminase; ALT, alanine aminotransferase; TBIL, total bilirubin; DBIL, direct bilirubin; PT, prothrombin time; APTT, activated partial thromboplastin time; AFP, alpha-fetoprotein; CEA, aspartate transaminase; SCC, Squamous cell carcinoma antigen; CYFRA2I-I, cytokeratin 19 fragment; TACE, transcatheter arterial chemoembolization.

TBIL and DBIL were negative. On admission, he had normal levels of AFP and CEA (Table 1). An abdominal CT scan conducted in a local hospital demonstrated a single, big and solid lesion with a diameter of $10 \mathrm{~cm}$, with mild to moderate enhancement in the parenchymal part of the tumor edge. There were no lesions in his lungs, gastrointestinal tract and other organs, indicating an initial disease with localized stage. At that time, a right hepatectomy with cholecystectomy was performed when a large lesion was found in the right lobe of the liver. The final pathology report revealed a diagnosis of primary SCC of the liver through routine $\mathrm{HE}$ (Figure 2A) and $\mathrm{IHC}$ analysis (Figure $2 \mathrm{~B}$ and $\mathrm{C}$ ), with chronic cholecystitis. There were no stones in the bile ducts and gallbladder. The negative staining of CK7 (Figure 2B), positive staining of p63 (Figure 2C), hepatocyte and CK19 indicated the squamous epithelium origin of the tumor. Positive PD-L1 staining on tumor cells but not on tumorassociated immune cells was observed (Figure 2D and E). This patient recovered well and was consistently treated with TACE and two cycles of adjuvant chemotherapy (gemcitabine combined with 5-fluorouracil). He did not undergo radiotherapy or other therapies. This patient has a long survival time of up to 35 months and still is alive until now.

\section{Case 3}

In July 2019, A 79-year-old woman came to our hospital and presented with abdominal pain, nausea, fever and weight loss over the past 3 months. She did not have a history of hepatitis virus infection, hepatic cyst, chronic cholecystitis or hepatolithiasis. Other medical history was negative. Hematological analysis revealed liver function was normal (Table 1). The levels of CEA and AFP were normal but serum ferroprotein and SCC antigen were slightly elevated. Enhanced magnetic resonance imaging (MRI) revealed a large mass in the right lobe of the liver $(7.5 \times 6.0 \times 5.6 \mathrm{~cm})$, with a long T1 and long T2 signal, a high signal in diffusion-weighted image (DWI), and a low signal in apparent diffusion coefficient (ADC). The lesions invaded the adjacent ascending colon and surrounding peritoneum (Figure 3A-C). A US-guided fineneedle aspiration biopsy of the mass confirmed a pathological diagnosis of SCC with a histological feature of keratinization (Figure 3D-F). IHC analysis indicated that the expression of PD-L1 on tumor cells was $30 \%$, and its expression on tumor-associated immune cells was $10 \%$ (Figure $3 \mathrm{G}$ and $\mathrm{H}$ ). Both chest $\mathrm{CT}$ and gastrointestinal radiography reported negative findings. Therefore, a final diagnosis of primary SCC of the liver was made. Unfortunately, due to the poor performance status, the patient only received tyrosine kinase inhibitors anlotinib monotherapy and died of dysfunctions of the liver and kidneys 2 months later.

\section{Primary SCC of the Liver from SEER Data}

There were 25 cases with primary SCC of the liver from the SEER database from 1997 to 2016. Baseline clinicopathologic features of these patients are shown in Table 2. 

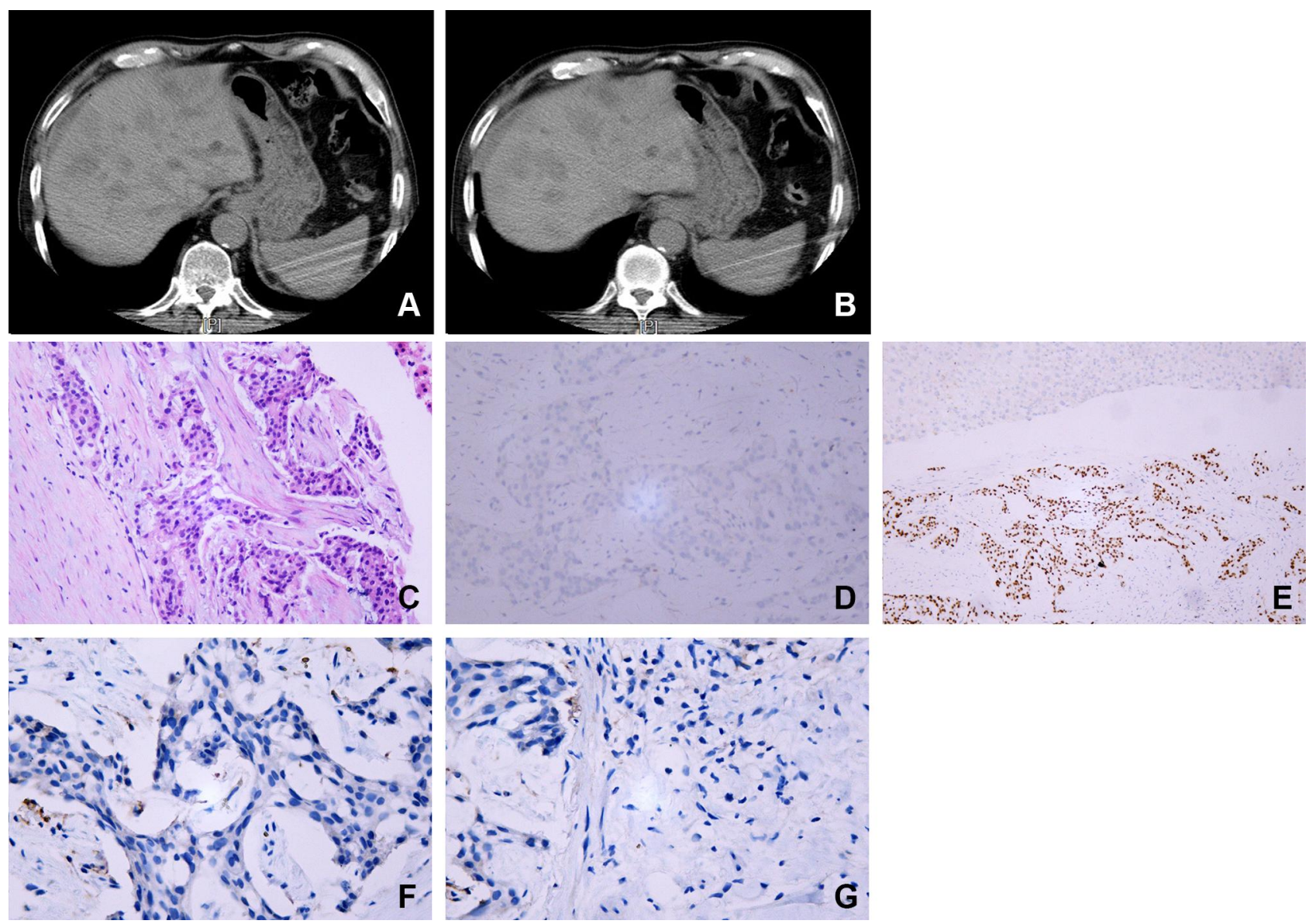

Figure I Radiographical findings and pathological reports for case I. CT scan of the abdomen revealed multiple low-density lesions in the liver accompanied by liver cysts (A and B). Pathological report revealed a diagnosis of SCC (C). IHC analysis showed that CK7 staining (D) was negative but p63 expression (E) was positive. Ventana IHC analysis (Clone NO. SP263) indicated that PD-LI expression on tumor cells and tumor-associated immune cells was negative (F and $\mathbf{G})$.

The mean and median age was 66.4 and 67.0 years (range 33-87 years), respectively. $56.0 \%$ of patients were male and $44.0 \%$ were female. $24.0 \%$ of patients received surgery and $32.0 \%$ received chemotherapy. The median OS (Figure 4A) and disease-specific survival (DSS) (Figure 4B) were 7.7 months (range 0.0-76.0 months) and 2.0 months (range 0.0-20.0 months), respectively. Furthermore, patients receiving surgery had a longer median OS (20.0 versus 6.0 months, $P=0.016$ ) (Figure 5A) and DSS (48.0 versus 8.0 months, $P=0.03$ ) (Figure 5B) than those receiving palliative treatment. Only five patients survived for more than one year. It is worth reminding that the overall survival time of a 55-year-old female patient with poorly differentiated SCC of the liver was 76 months.

\section{Discussion}

In this retrospective study, we reported three cases of primary SCC of the liver in a single center from 2013 to 2019 and analyzed their clinical and pathological characteristics, treatment and prognosis. We also reviewed all with the primary SCC of the liver from the SEER database from 1997 to 2016 and found that 25 patients with such disease had a median OS of 7.68 months and a median DSS of 2.00 months.

Previous studies showed that patients with primary SCC of the live presented with various symptoms including abdominal pain or discomfort, jaundice, loss of weight, loss of appetite, and rarely, progressive dysphagia. Palpable abdominal mass and tenderness over the right upper quadrant, with fever, are recognized as the most common initial signs. ${ }^{2}$ Radiographic analyses including enhanced CT and MRI help to determine the location of primary tumors, and also add useful information involving the number of lesions, their size, extent, degree of tumor invasion, whether biliary dilation occurs and whether all lesions are completely resected. Generally, the mass is mostly single, with unclear boundary, and its appearance is mostly round and lobulated. CT or MRI dynamic 

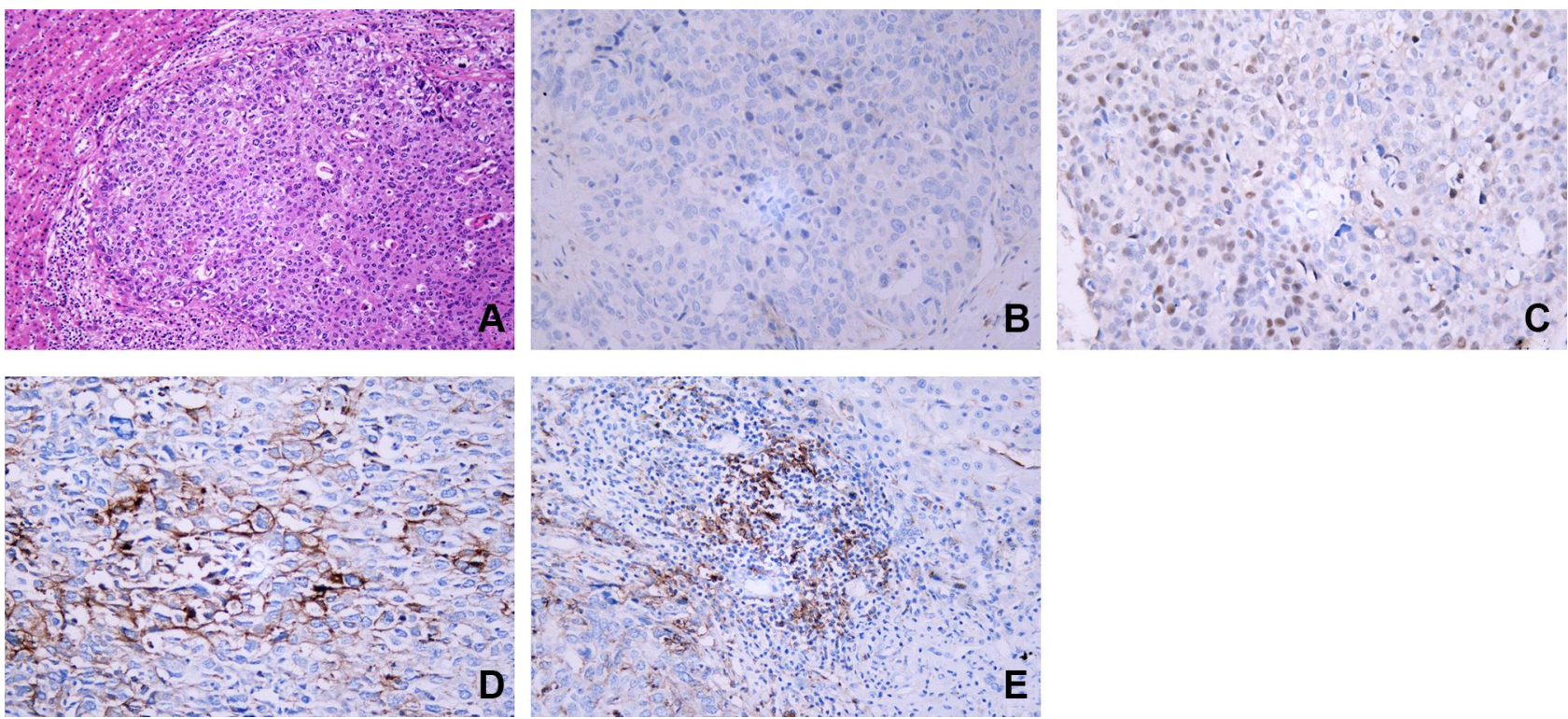

Figure 2 Pathological report revealed a diagnosis of SCC for case 2 (A). The IHC staining shows negative staining of CK7 (B) and the positive staining of P63 (C). PD-LI expression on tumor cells was 10\% (D), but its expression on tumor-associated immune cells was negative (E).
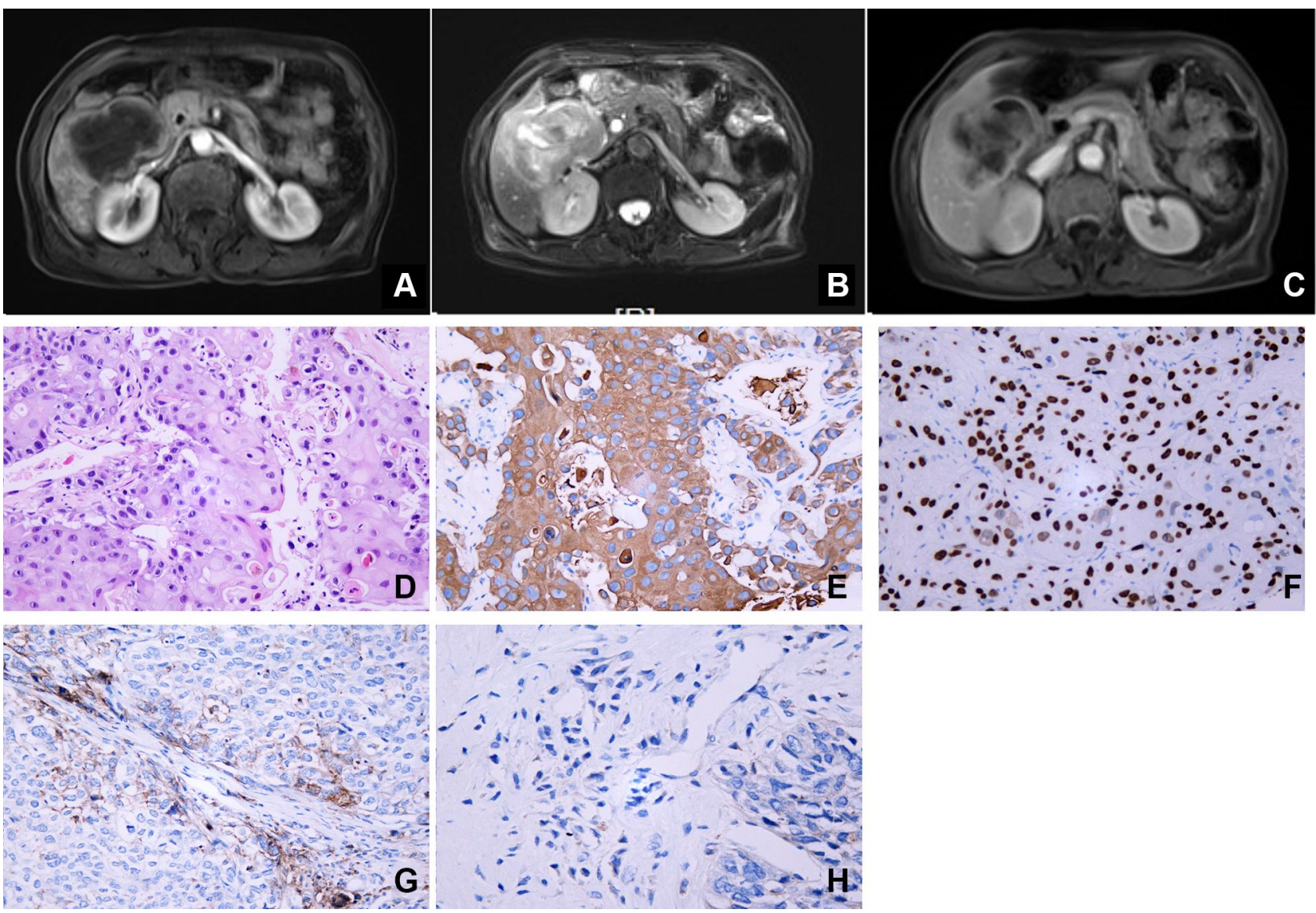

Figure 3 Radiographical findings and pathological reports for case 3. MRI revealed a large mass in the right lobe of the liver, with a long TI and long T2 signal, a high signal in DWI, and a low signal in ADC (A-C). Pathological report revealed a diagnosis of SCC (D). The IHC analysis showed positive staining of CK5/6 (E) and P63 (F). PD-LI expression on tumor cells was $30 \%(\mathbf{G})$, and its expression on tumor-associated immune cells was $10 \%(\mathbf{H})$. 
Table 2 Clinicopathologic Features of Primary SCC of the Liver in the SEER Database

\begin{tabular}{|c|c|}
\hline Characteristics & No. of Patients (\%) \\
\hline Age, years, mean $\pm S D$ & $66.4 \pm 13.2$ \\
\hline Age, years, median (range) & 67.0 \\
\hline \multicolumn{2}{|l|}{ Sex } \\
\hline Male & $14(56.0)$ \\
\hline Female & II (44.0) \\
\hline \multicolumn{2}{|l|}{ Age (year) } \\
\hline$\leq 60$ & $9(36.0)$ \\
\hline$>60$ & $16(64.0)$ \\
\hline \multicolumn{2}{|l|}{ Race } \\
\hline White & $14(56.0)$ \\
\hline Black & $6(24.0)$ \\
\hline Other & $5(20.0)$ \\
\hline \multicolumn{2}{|l|}{ AJCC TNM stage } \\
\hline 1 & $4(16.0)$ \\
\hline II & $2(8.0)$ \\
\hline III & I (4.0) \\
\hline IV & $5(20.0)$ \\
\hline Unknown & $13(52.0)$ \\
\hline \multicolumn{2}{|l|}{ Grade } \\
\hline 1 & I (4.0) \\
\hline II & $\mathrm{I}(4.0)$ \\
\hline III & $7(28.0)$ \\
\hline Unknown & $16(64.0)$ \\
\hline \multicolumn{2}{|l|}{ Surgery } \\
\hline No & $19(76.0)$ \\
\hline Yes & $6(24.0)$ \\
\hline \multicolumn{2}{|l|}{ Chemotherapy } \\
\hline No & $17(68.0)$ \\
\hline Yes & $8(32.0)$ \\
\hline \multicolumn{2}{|l|}{ Radiation } \\
\hline No & $25(100.0)$ \\
\hline Yes & $0(0.0)$ \\
\hline
\end{tabular}

contrast-enhanced scan showed mild to moderate enhancement in the parenchymal part of the tumor edge, and continuous enhancement in the portal vein phase. The central necrotic area is large and unenhanced, with uneven inner edges and sometimes nodular projections of different sizes; and tumor is generally not associated with chronic liver diseases such as cirrhosis. ${ }^{2}$ The diagnosis of primary
SCC of the liver is to exclude metastatic SCC of the liver from other primary sites, such as the lung, thyroid, esophagus, or gastrointestinal tract. In our cases, radiographic findings in major organs were negative and the tumors in the liver were diagnosed as primary. Primary SCC of the liver has been reported to be associated with hepatic cyst, chronic cholecystitis or hepatolithiasis in the majority of reported cases, ${ }^{8,11-20}$ and focal wall thickening of hepatic cysts or intrahepatic biliary from imaging might indicate a malignant change. However, its pathogenesis remains still uncertain. In our report, one case had a history of hepatic cysts and two cases were diagnosed with hepatolithiasis before or at the time of the confirmation of SCC of the liver. It is hypothesized that the SCC could develop from the ectopic squamous epithelium or from undifferentiated basal cells. Chronic inflammation of the bile duct or congenital cysts of the biliary tract or liver cysts associated with infection and/or stones has been attributed as the major etiological factors of malignant transformation. ${ }^{8,11-19}$ Continuous irritation due to chronic inflammation might be responsible for secondary squamous metaplasia and subsequent malignant transformation of SCC. ${ }^{8,11-19}$

Pain in the right upper region of the abdomen is the most frequently occurred symptom. However, this is not specific and should be distinguished from other primary liver or biliary tract diseases. In our cases, two patients were initially admitted with abdominal pain. In addition, altered liver function was usually observed in the primary SCC of the liver, including increased levels of ALT, AST, IBIL, and TBIL. In our reports, the second patient had increased levels of ALT and AST, but normal levels of BIL, and TBIL. Thus, the extent of liver dysfunction is associated with chronic inflammation of bile ducts, liver cyst, and cancer invasion or cancer-related obstruction of the bile duct. Unlike hepatocellular carcinoma, a specific serum tumor marker of primary SCC of the liver is not available. In our reports, all patients had normal levels of CEA and AFP, and only the second patient's serum ferroprotein and SCC antigen were slightly elevated.

Immunohistochemically, primary SCC of the liver is positive for $\mathrm{CK} 5 / 6$, p63, and $\mathrm{p} 40 .{ }^{2} \mathrm{CK} 19$ and $\mathrm{CK} 7$ can be used to mark the structure of glandular ducts, which can be used to differentiate primary SCC from the neoplastic cells of the bile ductular ontogeny. Hepatocellular carcinoma is characterized by arginase-1, GPC3, hepatocytes, AFP, and cholangiocellular carcinoma was positive for CK19 and CK8. In our reports, all patients were positive for p63 and 
A

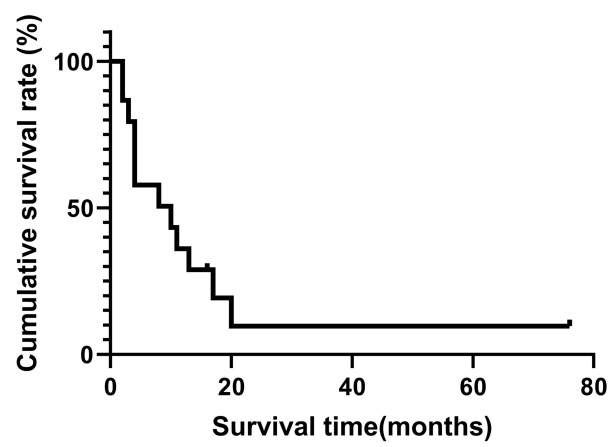

B

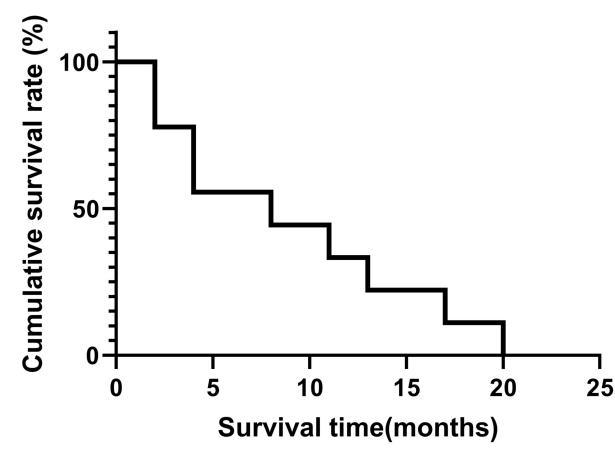

Figure 4 OS (A) and DSS (B) for all primary SCC of the liver from SEER database from 1997 to 2016.

negative for CK7, hepatocyte and CK19. The third patient was positive for $\mathrm{CK} 5 / 6$, indicating that basal cells of the keratinized squamous epithelium may be the origin of the cancer cells. Furthermore, we also excluded squamous cancer from other possible sites, including lungs, thyroid, gastrointestinal tract, other than the liver, through clinical, radiological, endoscopic, and endocrine detections.

At present, there is a lack of standard of care for primary SCC of the liver. As an aggressive cancer, the prognosis of this tumor is extremely unfavorable with an OS of less than 12 months. $^{2,6}$ As a rarely occurred cancer type, clinical trials associated with the treatment of this disease were not available. In previously reported cases, $42.1 \%$ of patients with surgery resection survived over 12 months. Patients with radical surgery treatment had longer overall survival compared to those with palliative treatment (median overall survival: 17 versus 5 months). ${ }^{2}$ In our reports, the second patient received radical surgery treatment and survived for 35 months. Therefore, complete surgical excision of the tumor, but not single drainage or partial excision is associated with long survival and should be recommended in the management of primary SCC of the liver. In addition, intrahepatoarterial chemotherapy and radiotherapy have been used in some patients who are not the candidates for surgery. Intrahepatoarterial chemotherapy with cisdiaminedichloro platinum and 5-fluorouracil led to a long survival time of 23 months in a primary liver SCC patient without surgery. ${ }^{8}$ Under certain circumstances, systematic chemotherapy is helpful for the treatment of advanced patients or acts as an adjuvant treatment before and after surgery. The second patient from our cohort received radical surgery treatment and survived for approximately 3 years. Interestingly, the first patient in our case series was not treated with surgery because of multiple lesions in the liver and received chemotherapy, with an OS of more than one year. Furthermore, we reported that the OS was less than 1 year for primary SCC of the liver from the SEER database from 1997 to 2016. To our knowledge, it is the first report of survival for a large number of primary liver SCC patients from SEER database. As an aggressive cancer, the prognosis of primary SCC of the liver is extremely unfavorable. In the SEER database, we found that only five patients survived for more than one year. Interestingly, patients receiving radical surgery had a longer median OS and
A

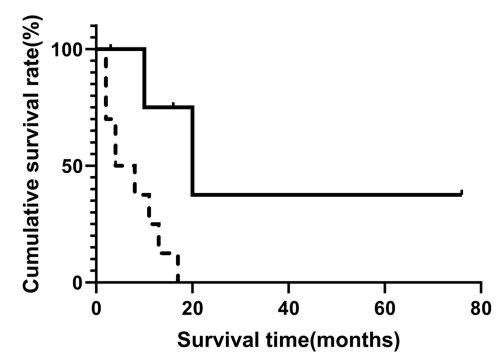

B

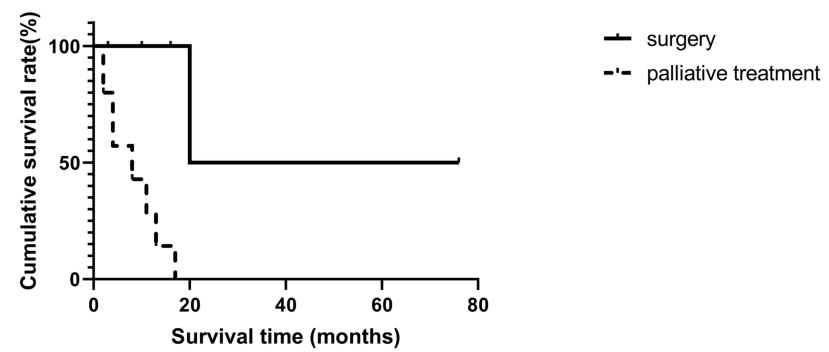

Figure 5 OS (A) and DSS (B) for primary SCC of the liver who received surgery or palliative treatment from SEER database from 1997 to 2016. 
DSS than those receiving palliative treatment. It is worth reminding that the overall survival time of a 55-year-old female patient with poorly differentiated SCC of the liver was 76 months. Thus, patients with this tumor should benefit from surgery if preoperative evaluation confirms that the tumor is surgically resectable. It is anticipated that systematic treatment can convert a selected group of patients with irresectable state into an operable state. Finally, immunotherapy with PD-1 or PD-L1 inhibitors has been approved for treating a variety of advanced human cancers, including lung squamous cell carcinoma, esophageal squamous carcinoma, cutaneous squamous cell carcinoma, and head and neck squamous cell carcinoma. ${ }^{20}$ Here we also found that two patients had positive tumor PD-L1 expression, and one patient had positive PD-L1 expression on immune cells, indicating that PD-1/PD-L1 signaling pathway may be activated. Although immunotherapy has not been a clinical therapeutic approach for primary liver SCC, PD-1/PD-L1 blockade by PD-1 inhibitors may be developed as potential agents by activating the host's anti-cancer immune response.

\section{Conclusion}

In summary, the incidence of primary liver SCC was extremely low. The causes of carcinogenesis and etiopathogenesis need to be further studied. The prognostic factors are still unclear. Radical surgery should be firstly recommended. Chemotherapy, arterial embolization, tyrosine kinase inhibitors, and immunotherapy are potential options for the treatment of primary SCC of the liver.

\section{Abbreviations}

SCC, squamous cell carcinoma; SEER, Surveillance, Epidemiology and End Results; TACE, transarterial chemotherapy and embolization.

\section{Acknowledgments}

Written informed consent for publication was provided by all patients, which was in accordance with the Helsinki Declaration. We sincerely thank the patients for their contribution to the publication of this case series.

\section{Disclosure}

The authors report no conflicts of interest in this work.

\section{References}

1. Banbury J, Conlon KC, Ghossein R, Brennan MF. Primary squamous cell carcinoma within a solitary nonparasitic hepatic cyst. J Surg Oncol. 1994;57(3):210-212. doi:10.1002/jso.2930570316

2. Zhang XF, Du ZQ, Liu XM, Lv Y. Primary squamous cell carcinoma of liver: case series and review of literatures. Medicine (Baltimore). 2015;94(28):e868. doi:10.1097/MD.0000000000000868

3. Weimann A, Klempnauer J, Gebel M, et al. Squamous cell carcinoma of the liver originating from a solitary non-parasitic cyst case report and review of the literature. HPB Surg. 1996;10(1):45-49. doi:10.1155/1996/97680

4. Iimuro Y, Asano Y, Suzumura K, et al. Primary squamous cell carcinoma of the liver: an uncommon finding in contrast-enhanced ultrasonography imaging. Case Rep Gastroenterol. 2011;5 (3):628-635. doi:10.1159/000334425

5. Spaggiari M, Di Benedetto F, Ballarin R, et al. Primary squamous cell carcinoma of the liver associated with Caroli's disease: a case report. Onkologie. 2011;34(4):193-195. doi:10.1159/000326999

6. Zhao R, Zhu K, Wang R, et al. Primary squamous cell carcinoma of the liver: a case report and review of the literature. Oncol Lett. 2012;4 (6):1163-1166. doi:10.3892/ol.2012.894

7. Zhu KL, Li DY, Jiang CB. Primary squamous cell carcinoma of the liver associated with hepatolithiasis: a case report. World $J$ Gastroenterol. 2012;18(40):5830-5832. doi:10.3748/wjg.v18. i 40.5830

8. Kaji R, Sasaki N, Tateishi I, et al. A case report of primary hepatic squamous cell carcinoma that remarkably responded to low dose arterial injection of anti-cancer drugs. Kurume Med J. 2003;50(1/ 2):71-75. doi:10.2739/kurumemedj.50.71

9. Naik S, Waris W, Carmosino L, Mehrishi A, Saif MW. Primary squamous cell carcinoma of the liver. $J$ Gastrointestin Liver Dis. 2009;18(4):487-489.

10. Morito K, Kai K, Miyoshi A, et al. Primary squamous cell carcinoma of the liver concomitant with primary colon cancer: report of a case. Clin J Gastroenterol. 2013;6(2):134-138. doi:10.1007/s12328-0120341-2

11. Nieweg O, Slooff MJ, Grond J. A case of primary squamous cell carcinoma of the liver arising in a solitary cyst. HPB Surg. 1992;5 (3):203-208. doi:10.1155/1992/32474

12. Boscolo G, Jirillo A, Da Pian P. Complete remission of poorly differentiated squamous liver carcinoma after systemic chemotherapy and surgery: a case report. Tumori. 2005;91(1):71-72. doi:10.1177/ 030089160509100113

13. Roediger WE, Dymock RB. Primary squamous carcinoma of the liver: clinical and histopathological features. Aust $N Z J$ Surg. 1991;61(9):720-722. doi:10.1111/j.1445-2197.1991.tb00331.x

14. Yagi H, Ueda M, Kawachi S, et al. Squamous cell carcinoma of the liver originating from non-parasitic cysts after a 15-year follow-up. Eur J Gastroenterol Hepatol. 2004;16(10):1051-1056. doi:10.1097/ 00042737-200410000-00016

15. Clements D, Newman P, Etherington R, Lawrie BW, Rhodes J. Squamous carcinoma in the liver. Gut. 1990;31(11):1333-1334. doi:10.1136/gut.31.11.1333

16. Bloustein PA, Silverberg SG. Squamous cell carcinoma originating in an hepatic cyst. Case report with a review of the hepatic cyst carcinoma association. Cancer. 1976;38(5):2002-2005. doi:10.1002/1097-0142(197611)38:5<2002::AIDCNCR2820380523>3.0.CO;2-9

17. Lynch MJ, Mcleod MK, Weatherbee L, Gilsdorf JR, Guice KS, Eckhauser FE. Squamous cell cancer of the liver arising from a solitary benign nonparasitic hepatic cyst. Am J Gastroenterol. 1988;83(4):426-431. 
18. Monteagudo M, Vidal G, Moreno M, et al. Squamous cell carcinoma and infection in a solitary hepatic cyst. Eur J Gastroenterol Hepatol. 1998;10(12):1051-1053. doi:10.1097/00042737-199812000-00012

19. Vick DJ, Goodman ZD, Ishak KG. Squamous cell carcinoma arising in a ciliated hepatic foregut cyst. Arch Pathol Lab Med. 1999;123 (11):1115-1117. doi:10.1043/0003-9985(1999)123<1115:SCCAIA $>2.0$. $\mathrm{CO} ; 2$
20. Zhang B, Song Y, Fu Y, Zhu B, Wang B, Wang J. Current status of the clinical use of PD-1/PD-L1 inhibitors: a questionnaire survey of oncologists in China. BMC Cancer. 2020;20(1):86. doi:10.1186/ s12885-020-6583-3

\section{Publish your work in this journal}

Cancer Management and Research is an international, peer-reviewed open access journal focusing on cancer research and the optimal use of preventative and integrated treatment interventions to achieve improved outcomes, enhanced survival and quality of life for the cancer patient.
The manuscript management system is completely online and includes a very quick and fair peer-review system, which is all easy to use. Visit http://www.dovepress.com/testimonials.php to read real quotes from published authors. 